\title{
Emotional Impact for Predicting Student Performance in Intelligent Tutoring Systems (ITS)
}

\author{
Kouamé Abel Assielou ${ }^{1}$, Cissé Théodore Haba ${ }^{2}$ \\ Bi Tra Gooré ${ }^{3}$, Tanon Lambert Kadjo ${ }^{4}$ \\ Dept. Computer Science Research and Telecommunication \\ (LARIT), Institut National Polytechnique Felix \\ HOUPHOUET Boigny (INP-HB) \\ Yamoussoukro, Côte d'Ivoire
}

\author{
Kouakou Daniel Yao \\ Dept. of Studies and Prevention in Psychoeducation \\ (LEPPE-ENS) \\ University Jean Lorougnon Guédé \\ Daloa, Côte d'Ivoire
}

\begin{abstract}
Current Intelligent Tutoring Systems (ITS) provide better recommendations for students to improve their learning. These recommendations mainly involve students' performance prediction, which remains problematic for ITS, despite the significant improvements made by prediction methods such as Matrix Factorization (MF). The present contribution therefore aims to provide a solution to this prediction problem by proposing an approach that combines Multiple Linear Regression (Modelling Emotional Impact) and a Weighted MultiRelational Matrix Factorization model to take advantage of both student cognitive and emotional faculties. This approach takes into account not only the relationships that exist between students, tasks and skills, but also students' emotions. Experimental results on a set of pedagogical data collected from 250 students show that our approach significantly improves the results of Student Performance Prediction.
\end{abstract}

Keywords-Intelligent tutoring system; student performance prediction; matrix factorization; emotional impact; achievement emotions

\section{INTRODUCTION}

Computer learning systems, in particular Intelligent Tutoring Systems occupy a preponderant place today. These systems are IT environments that aim to best imitate the behavior of a human tutor in his capacity as a field expert and educational expert. Current researches are based on some domains such as student modelling in general and student performance prediction in particular. Let's mention that the student performance prediction in learning systems remains a major research issue because it predicts its performance for different skills over time [1]. In addition, it makes it possible to determine the next content to be presented to the student to give continuity to his learning [2]. Indeed, in the absence of a good prediction, presenting students with activities that are too easy for example can limit learning on the one hand, and on the other hand, presenting difficult activities can also lead to a misunderstanding and demotivation that are too strenuous also risk leading to poor understanding and loss of motivation [3].

Therefore, for student adequate learning, it is important that current systems have relevant information or attributes (characteristics) to refine the results of performance prediction. Besides, for a better understanding of the educational paths of students, it is useful not to limit information taking only to cognitive or economic areas, but to also have information on the emotional state of students. The emotion, not being always taken into account in the systems of prediction, it generally leads to less efficient systems. Indeed, for specialists in Educational Psychology and neuroscience such as Immordino-Yang [4], emotions are almost essential at the neurobiological level to create memories, engage complex thoughts or even come to significant decisions. They are important for our physical survival as well as our social or intellectual life [4]. Giving systems the ability to integrate emotional aspect will allow them to better understand the student's needs and to react accordingly. This is, moreover, the objective of the research area in affective computing [5] [6]. Our objective is therefore to provide our model with significant data such as emotion to increase the quality of performance prediction.

This paper is organized according to the following order: Section 2 provides a State-of-The-Art of techniques and attributes used to predict student performance. Section 3 in turn presents classic Matrix Factorization techniques and the emotional data acquisition method used, while Section 4 describes our Multi-Relational and Emotional approach to predicting student performance. Section 5 presents an evaluation of this approach and provides a comparative study of our results with those of State-of-The-Art. Section 6 concludes this paper.

\section{STATE-OF-THE-ART}

Personalized learning improves not only the learning process itself but also the overall learning outcomes [7] [8]. But this personalization is only possible by adapting the content of the learning and applying a learning strategy adapted to each individual. Also, with regard to current ITS, it is necessary to have, on the one hand, relevant information on student and, on the other hand, to predict his performance at each stage of his learning. A great deal of research on student performance predicting is available in the associated scientific literature. Student performance prediction is carried out according to several attributes and methods. The choice of selected attributes and the choice of methods used are decisive for predicting performance, in precise evaluation frameworks. In [9] [10] [11], for example, authors chose attributes such as internal marks, session marks, admission score and students' activities by applying decision trees. Raheel Asif et al., [10] showed that using this approach, we could achieve a 
performance prediction accuracy of around $83.65 \%$. Other authors such as Abu Amra et al., [12] extended the choice of attributes to the student's father work, the student's place of residence, the gender, and the past performances by applying two different types of classification algorithms: K-Nearest Neighbors (KNN) and Naïve Bayes. Experimental results have shown that the Naïve Bayes method is better than the KNN method with an accuracy of 93.6\%. Okubo et al., [13] proposed a Recurrent Neural Network (RNN) approach to predict students' final grades from journal data stored in education systems.

Besides, several other studies such as that carried out in [14] [15] [16] rather propose methods of Data Mining making it possible to search for attributes which significantly affect student performance. Shana et al., [14] for example have extracted, by applying two statistical techniques which are Pearson correlation coefficient and Fisher test, attributes such as previous school marks, level of difficulty of the subject, income family and the student's interest in the subject. Havan Agrawal et al., [15] showed on the basis of a Neural Network model that the current students' academic performances depends mainly on their past performances. The authors Haixia Lu et al., [16] proposed an algorithm for characteristics (attributes) selection which uses Pearson correlation coefficient and Euclidean Distance to measure the correlation between characteristics. The selected characteristics include, among others: past marks, number of school absences, student's willingness to pursue higher education, student age, number of past failures. In [17] the authors compare the results of the two classifiers Support Vector Machine (SVM) and KNN on the data set provided by the University of Minho in Portugal, in order to predict student's final grade. The data set concerns students' mathematical performance and consists of 395 data samples. Characteristics selection was done by calculating the correlation between each attribute (33 Attributes in all) and the target variable (which is the final score). Empirical studies results have shown that the SVM algorithm performs slightly better than the KNN algorithm. The author Seifedine Kadry [18] has shown through a linear regression analysis that the independent variables studied: height, weight, IQ score, sex and age of a student have no significant impact on the performance of it. But the revision time has a significant effect on his performance on the exam.

Other studies such as those conducted in [19] [20] have used Matrix Factorization, a technique derived from recommendation systems to predict students' performance. The results of these studies have shown that the use of this technique can improve prediction results compared to regression methods by building on the student's past performance. Other authors have proposed improving this method by exploring multiple relationships that may exist between students, tasks and their metadata using MultiRelational Matrix Factorization (MRMF) and Weighted MultiRelational Matrix Factorization (WMRMF) models [21]. In [22] we proposed a Social and Weighted Multi-Relational Matrix Factorization model which integrates not only friendship relationships like group work relationships, but also mutual influence values to favor error reduction in student performance prediction.
It is clear from this literature review that the majority of research has relied mainly on socio-economic factors and on student past skills without taking into account of emotional aspect. That is constitutes the object of our present study.

\section{METHODOLOGICAL APPROACH}

Our approach combines a Weighted Multi-Relational Matrix Factorization model and a Multiple Linear Regression modelling Emotional Impact. Therefore, this session presents some Matrix Factorization approaches as methods that we will use to describe and compare our proposed approach. In addition, it presents the method of self-assessment of emotions through the concept of Achievement Emotions.

\section{A. Matrix Factorization Approachs}

1) Classical Matrix Factorization (MF) approach: Consider a set $S$ of students, a tasks set $I$, and a range of possible performance scores $P$. Matrix Factorization consists in approximating a matrix $R$ by a product of two matrices $W_{1}$ (Students) and $W_{2}$ (Tasks) [22] [23] [24] as described by (1)

$$
R \approx W_{1} W_{2}^{T}
$$

$W_{1} \in \mathbb{R}^{S \times F}$ is a matrix where each line $s$ is a vector containing the $F$ latent factors describing the student $s$ and $W_{2} \in \mathbb{R}^{I \times F}$ is a matrix where each line $i$ is a vector containing the $F$ latent factors describing the task $i$. Let $w_{1_{s}}$ and $w_{2_{i}}$ be the respective vectors of the matrices $W_{1}$ and $W_{2}$ such that their elements are designated by $w_{1_{s f}}$ and $w_{2_{i f}}$.

In this method, a student $s$ performance in accomplishing a task $i$ is predicted by (2) :

$$
\hat{p}_{s i}=\sum_{f=1}^{F} w_{1_{s f}} w_{2_{i f}}=w_{1_{s}} w_{2_{i}}^{T}
$$

$\hat{p}_{s i}$ is the predicted performance value.

$W_{1}$ and $W_{2}$ designate the model parameters and can be learned by optimizing (3) using Stochastic Gradient Descent method as suggested in [25].

$$
O^{M F}=\sum_{(s, i) \in R}\left(R_{s i}-w_{1_{s}} w_{2_{i}}^{T}\right)^{2}+\lambda\left(\left\|W_{1}\right\|_{F}^{2}+\left\|W_{2}\right\|_{F}^{2}\right)
$$

The symbol $\|\cdot\|_{F}^{2}$ indicates the Frobenius norm and $\lambda$ is a regulation parameter [26]. Let $e_{s i}$ denote the difference between the real performance value and the predicted performance value for each couple (Student, Task). the prediction error is given by (4) :

$$
e_{s i}=\left(R_{s i}-w_{1_{s}} w_{2_{i}}^{T}\right)
$$

2) Multi-Relational Matrix Factorization (MRMF) approach: Unlike the MF model, the MRMF model refers to 
$N$ types of entities $\left\{E_{1}, \ldots, E_{N}\right\}$ connected by $M$ types of relationships $\left\{R_{1}, \ldots, R_{M}\right\}$ that can be strongly correlated with each other. Let $W_{1}, W_{2}, \ldots, W_{n}(n \in N)$, be the latent factor matrices (designating the parameters of the model) of each of entity types. Equation (3) thus becomes (5) [26] [21] [27]:

$O^{M R M F}=\sum_{r=1}^{M} \sum_{(s, i) \in R_{r}}\left(R_{r_{s i}}-w_{r 1_{s}} w_{r 2_{i}}^{T}\right)^{2}+\lambda\left(\sum_{n=1}^{N}\left\|W_{n}\right\|_{F}^{2}\right)$

With $R_{r}=\left\{\left(E_{1 r} ; E_{2 r}\right)\right\}(r=1 \ldots M)$

3) Weighted Multi-Relational Matrix Factorization approach (WMRMF): The WMRMF model incorporates a weight factor which gives the importance of one relation compared to another.

Equation (5) thus becomes (6) [21] [27] [28]:

$o^{W M R M F}=\sum_{r=1}^{M} \Theta_{r} \sum_{(s, i) \in R_{r}}\left(R_{r_{s i}}-w_{r 1_{s}} w_{r 2_{i}}^{T}\right)^{2}+\lambda\left(\sum_{n=1}^{N}\left\|W_{n}\right\|_{F}^{2}\right)$

With: $\Theta_{r}=\left\{\begin{array}{l}1, \text { if } r \text { is the main relation } \\ \theta, \text { si }(0<\theta<1)\end{array}\right.$

The model parameters are updated through (8) and (9).

$w_{r 1_{s k}}^{\prime}=w_{r 1_{s k}}+\beta\left(2 \Theta_{r} e_{r_{s i}} w_{r 2_{i k}}-\lambda w_{r 1_{s k}}\right)$

$w_{r 2_{i k}}^{\prime}=w_{r 2_{i k}}+\beta\left(2 \Theta_{r} e_{r_{s i}} w_{r 1_{s k}}-\lambda w_{r 2_{i k}}\right)$

The parameter $\beta$ designates the learning rate.

\section{B. Achievement Emotions}

In our Multi-Relational approach, we suggest taking into account the possible emotions felt by students during the evaluation sessions. The consideration of emotions suggests, beforehand, the choice of a category of candidate emotions and the choice of a method of emotional detection.

In context of emotions candidate selection, several research studies have generally focused on those related to learning but in particular on those related to learning evaluation [29] [30]. The aim of this work was, through theoretical and statistical studies, to research different emotions that are aroused in a learning context.

In this study, we based on Pekrun's [31] control-value theory of achievement emotions.

The theory proposed by Pekrun strives to provide an integration framework for analyzing the antecedents and effects of emotions experienced in achievements in an academic environment. These emotions are also called achievement emotions. They occur in different academic contexts such as taking courses, studying and taking tests / exams [32]. Achievement emotions are considered to be emotions directly related to achievement activities and / or achievement outcomes [33] [29]. These activities refer to tests, assignments and performances both in examination conditions and as mere tasks, whereas achievement outcomes refer to for instance grades and scores [34]. Empirical evidence from Pekrun's theory indicates that achievement emotions have three dimensions [34]:

- Valence (positive / pleasant versus negative / unpleasant);

- Activation (activation versus deactivation);

- Focus on the object (activity versus outcome).

The control-value theory of achievement emotions considers the emotions like sets of interdependent psychological processes, according to which the affective, cognitive, motivational and physiological components are of first importance [32].

In our approach, due to the complexity of emotion detection linked to the test, we used the self-assessment method based on Achievement Emotions Questionnaire (AEQ) to detect students' emotions. The AEQ was developed by Pekrun and his colleagues [32]. It is a self-assessment tool for measuring students' emotions in school situations. The instrument, in its final version, consists of 24 emotion scales which are organized into three sections evaluating both classrelated, learning and test-related emotions [32]. The AEQ is made up of 232 items that measure eight different emotions related to the class, eight emotions related to studying, and eight emotions to writing tests and exams. A 5-point Likert scale ( 1 for completely disagree and 5 for completely agree) is used to assess the emotional experiences of students. The emotional scales related to the test include 77 items and the instruction asks students to indicate what they feel in terms of enjoyment, hope, pride, relief, anger, anxiety, shame, and hopelessness related to the test. These eight emotional scales are those chosen by our approach.

\section{PRoposed PREDICTION APPROACH}

In this section, we present our approach or predicting student performance that takes into account student's emotional state. Fig. 1 presents, in entity-association diagram form, the information taken into account by our model.

\section{A. Problem Formulation}

The student performs a task by associating a performance score and the emotions associated with the execution of the task (emotions recovered using the AEQ).

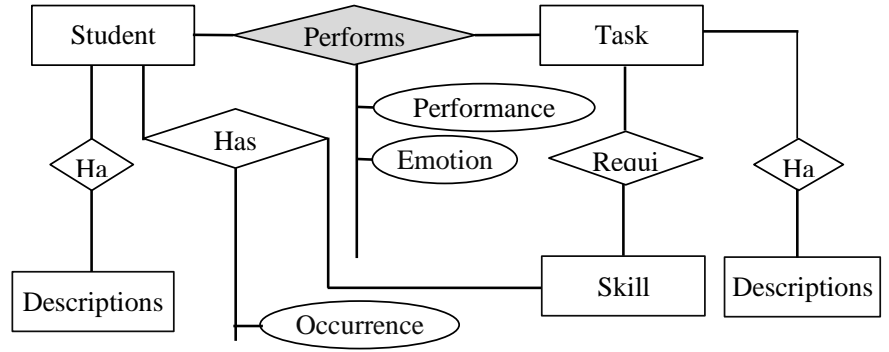

Fig. 1. Entity-Association Diagram for Predicting Student Performance.

Solving correctly the task, student must know specific skills, and task itself is also associated with the skills to be 
learned by student. The attribute "Occurrence" gives the number of times student has learned the skill.

\section{B. Emotional Influence Formulation $\varepsilon_{s i}$}

We hypothesize that any task accomplishment is always accompanied by one or more well-given emotions. We adopt the principle that a student's performance depends not only on their intrinsic abilities (cognitive abilities), but also on his emotions experienced impact.

Note $\varepsilon_{s i}$, the emotional impact on the student's performance in completing task $i$. In our approach, $\varepsilon_{s i}$ is defined as a Multiple Linear Regression model which establishes a linear relationship with the different test emotions of AEQ, relative to task $i$. The different values taken by $\varepsilon_{s i}$ are reals included in interval $[-1 ; 1]$. The different coefficients are elements of matrix $\alpha=\left(\alpha_{1}, \alpha_{2}, \ldots, \alpha_{8}\right)$. The different variables are elements of matrix $e=\left(e_{1}, e_{2}, \ldots, e_{8}\right)^{T}$. These elements are respectively the intensities of the eight scales of emotions linked to the test which are: enjoyment, hope, pride, relief, anger, anxiety, shame and hopelessness, and are collected using of the AEQ. Table I presents the different variables and descriptions of variables as proposed.

The different values $e_{1}, e_{2}, \ldots, e_{8}$ are easily obtained using AEQ. So, we formulate model $\varepsilon_{s i}$ as follows:

$$
\varepsilon=\alpha \cdot e+\alpha_{0}
$$

The model $\varepsilon_{s i}$ was developed on the basis of data collected during the AEQ survey. Unlike the work done in [21], we consider emotion as an additional entity type. Indeed, the relation «Student - Performs - Task » does not only take into account performance, but also the possible associated emotions and their valences (Fig. 2).

This relationship generates two matrices: the performance matrix and the emotional impact matrix. The emotional impact matrix has the same dimension as the performance matrix as shown in the example in Fig. 3. In this example, we notice that student $\mathrm{S}_{4}$ performed task $\mathrm{T}_{5}$ by obtaining the score of 1 . The emotional impact factor on performance, on task $\mathrm{T}_{5}$ completion is $\varepsilon_{45}=0.8$. We consider this value as the contribution of emotions to task $\mathrm{T}_{5}$ achievement. This contribution can either favor task accomplishment, or disadvantage it.

TABLE I. VARIABLES DESCRIPTION IN MODEL $\varepsilon_{s i}$

\begin{tabular}{|c|c|c|c|c|c|c|c|c|}
\hline Variables & $e_{1}$ & $e_{2}$ & $e_{3}$ & $e_{4}$ & $e_{5}$ & $e_{6}$ & $e_{7}$ & $e_{8}$ \\
\hline Intensity of : & 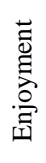 & 蒙 & $\frac{\pi}{2}$ & 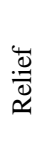 & 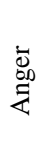 & $\begin{array}{l}\stackrel{\vec{d}}{\frac{d}{x}} \\
\frac{0}{2}\end{array}$ & $\begin{array}{l}\stackrel{\mathscr{E}}{\tilde{\Xi}} \\
\text { }\end{array}$ & 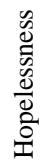 \\
\hline
\end{tabular}

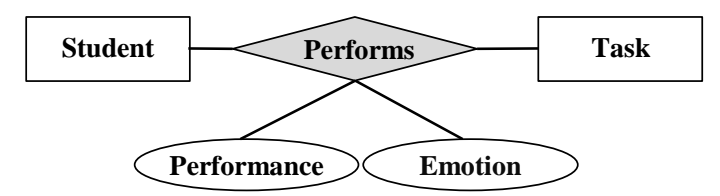

Fig. 2. Relation « Student - Performs - Task ».

In our approach, we combine model $\varepsilon_{s i}$ with the MRMF and WMRMF models, through the iterative algorithm 1.

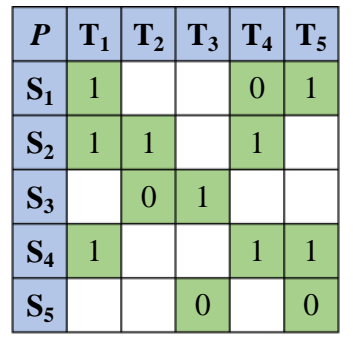

\begin{tabular}{|l|l|l|l|l|l|}
\hline$\varepsilon$ & $\mathbf{T}_{\mathbf{1}}$ & $\mathbf{T}_{\mathbf{2}}$ & $\mathbf{T}_{\mathbf{3}}$ & $\mathbf{T}_{\mathbf{4}}$ & $\mathbf{T}_{\mathbf{5}}$ \\
\hline $\mathbf{S}_{\mathbf{1}}$ & 0.7 & & & 0.4 & 0.7 \\
\hline $\mathbf{S}_{\mathbf{2}}$ & 0.6 & 0.9 & & 0.9 & \\
\hline $\mathbf{S}_{\mathbf{3}}$ & & 0.3 & 0.5 & & \\
\hline $\mathbf{S}_{\mathbf{4}}$ & 0.7 & & & 0.7 & 0.8 \\
\hline $\mathbf{S}_{\mathbf{5}}$ & & & 0.9 & & 0.1 \\
\hline
\end{tabular}

Fig. 3. Performance Matrix (P) and Emotional Impact Matrix $(\varepsilon)$

To determine optimal parameters $W_{1}, W_{2}, \ldots, W_{n}$ of this approach, we proposed the iterative algorithm 1 below using a Stochastic Gradient Descent.

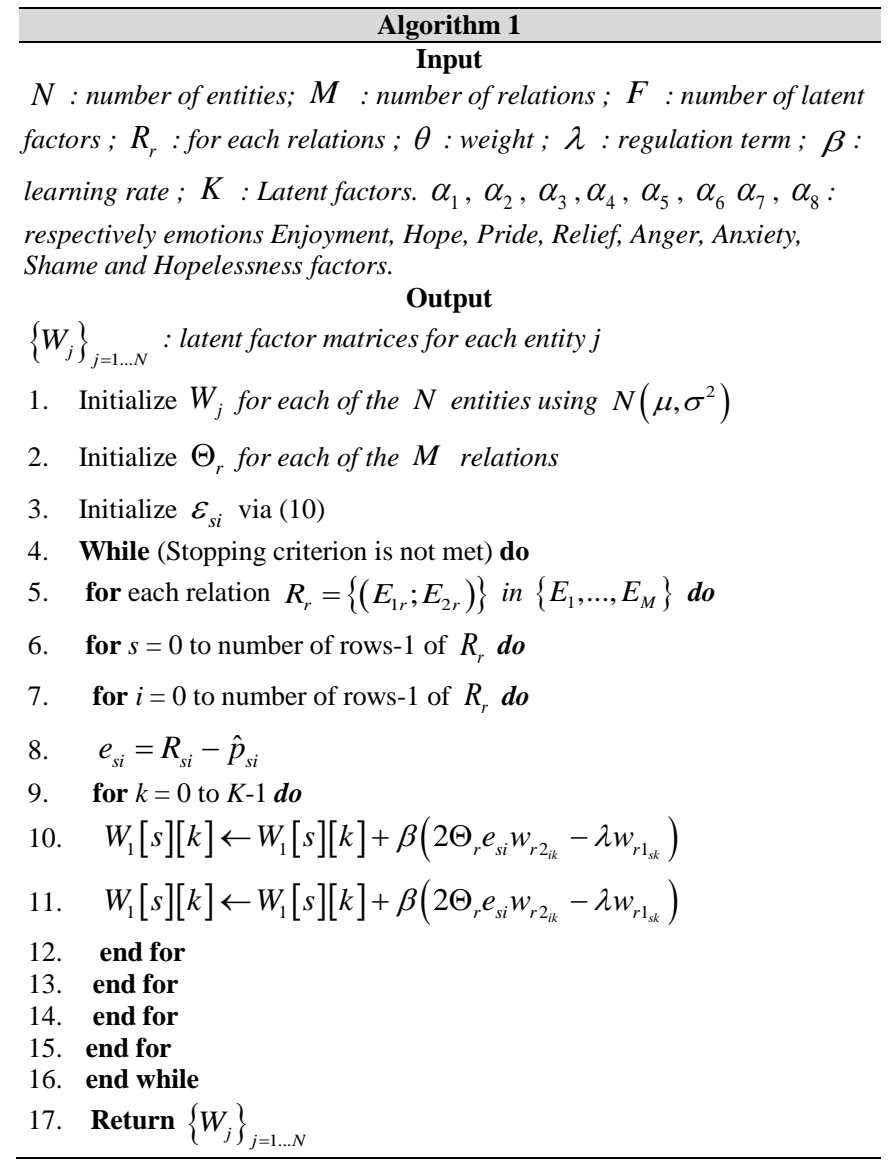

This algorithm proceeds first by initializing the parameters from the normal distribution $N\left(\mu, \sigma^{2}\right)$, taking for expectation $\mu=0$ and for standard deviation $\sigma=0.01$. Numerical 
simulation through Algorithm 1 also makes it possible to determine the optimal values of matrix $\alpha=\left(\alpha_{1}, \alpha_{2}, \ldots, \alpha_{8}\right)^{T}$ parameters. These values are also those which minimize (6).

\section{Evaluation OF Proposed APPROACH}

In this section, we evaluate our proposed approach in order to show its effectiveness in reducing errors in student performance prediction. We also perform tests to compare our approach performance with certain methods in the literature.

\section{A. Participants and Procedure}

To evaluate our emotional modelling approach and study the effect of taking emotion into account in student performance prediction, we carried out studies on upper secondary learners of general education, enrolled in class of Terminal at Lycée Moderne Khalil (Daloa, Côte d'Ivoire). The data were collected by means of the questionnaire distributed to the voluntary student to participate in this research. The sample consisted of 250 students (80 girls with an average age of 16; and 170 boys with an average age of 17). These students were assessed during exams in several disciplines: Mathematics, French, English, History and Geography, Philosophy and Biology Science, for the 2019 - 2020 school year from January to February. The questionnaire was filled out several times for each test in the exam. For our model evaluation, we used the eight emotion scales linked to the AEQ tests. These scales are composed of four emotions with positive valence and four emotions with negative valence.

\section{B. Results and Discussion}

We implement two types of models. The first is a Multi Relational and Emotional model noted Emo-MRMF and the second is a Weighted Multi Relational and Emotional model noted Emo-WMRMF. For the evaluation, we reduced the model $\varepsilon_{s i}$ to three scales of test emotions: Enjoyment, Hope and Anxiety. Indeed, positive activating emotions such as enjoyment and hope can lead to better performance results [35] while negative emotions such as anxiety can lead to poorer performance results when triggered [36]. In [30] [37] the authors have shown that positive emotions such as enjoyment and hope are generally positively correlated with test scores and results. The scale model looks like this:

$\varepsilon_{s i}=\alpha_{0}+\alpha_{1} e_{1}+\alpha_{2} e_{2}+\alpha_{6} e_{6}$

The working environment used for simulations is a 64-bit operating system computer, 8GB of RAM, intel Core i7. The Algorithm 1 development was done in Python language.

To compare our Emo-MRMF and Emo-WMRMF approaches to the MF, MRMF, WMRMF, Global Average, Item Average and User Average methods, we used the RMSE (Root Mean Squared Error) metric. This metric is calculated as follows:

$R M S E=\sqrt{\frac{\sum_{(r, s, i) \in D^{t e s t}}\left(p_{s i}-\hat{p}_{s i}\right)^{2}}{\left|D^{t e s t}\right|}}$
For referencing, we report the best hyperparameters that we found via cross-validation in Table II.

The experimental results presented in Fig. 4 show that our Emo-MRMF and Emo-WMRMF approaches significantly improve the MFMF and WMRMF approaches by taking into account multiple relationships and the emotional impact factor. The Emo-WMRMF model gives very good results in terms of RMSE reduction compared to simulated comparative methods. Our results show the importance of taking into account emotions in the field of learning in general and in particular in the prediction of student performance. These results also show that emotions have an influence on cognitive abilities such as problem solving and decision making as suggested by Lois Isenman [38]. These results demonstrate the robustness of Multi Relational Matrix Factorization models to ingest a variety of information and relationships.

TABLE II. OPTIMIZING HYPERPARAMETERS

\begin{tabular}{|l|l|}
\hline Methods & Parameter \\
\hline MF & $K=2 ; \#$ iter $=320 ; \beta=10^{-3} ; \lambda=0.15$ \\
\hline MRMF & $K=2 ; \#$ iter $=320 ; \beta=10^{-3} ; \lambda=0.1 ;$ \\
\hline WMRMF & $K=2 ; \#$ iter $=320 ; \beta=3.10^{-3} ; \lambda=75.10^{-4} ;$ \\
& $\theta \in\{1.0 ; 0.80 ; 0.70\}$ \\
\hline Emo - & $K=2 ; \#$ iter $=320 ; \beta=10^{-2} ; \lambda=55.10^{-3} ;$ \\
MRMF & $\alpha_{1}=14.10^{-3} ; \alpha_{2}=22.10^{-3} ; \alpha_{3}=-33.10^{-3}, \alpha_{0}=0$ \\
\hline & $K=2 ; \#$ iter $=320 ; \beta=3.10^{-3} ; \lambda=15.10^{-4} ;$ \\
Emo- & $\theta \in\{1.0 ; 0.40 ; 0.80 ; 0.70\} ; \alpha_{1}=3.10^{-3} ; \alpha_{2}=2.10^{-3} ;$ \\
WMRMF & $\alpha_{3}=-3.10^{-3} ; \alpha_{0}=0$ \\
\hline & $K=2 ; \#$ iter $=320 ; \beta=3.10^{-3} ; \lambda=15.10^{-4} ;$ \\
Emotional & $\theta \in\{0.7 ; 1.0 ; 0.60 ; 0.40\} ; \alpha_{1}=5.10^{-3} ; \alpha_{2}=25.10^{-3} ;$ \\
Impact & $\alpha_{3}=-23.10^{-3}$ \\
\hline
\end{tabular}

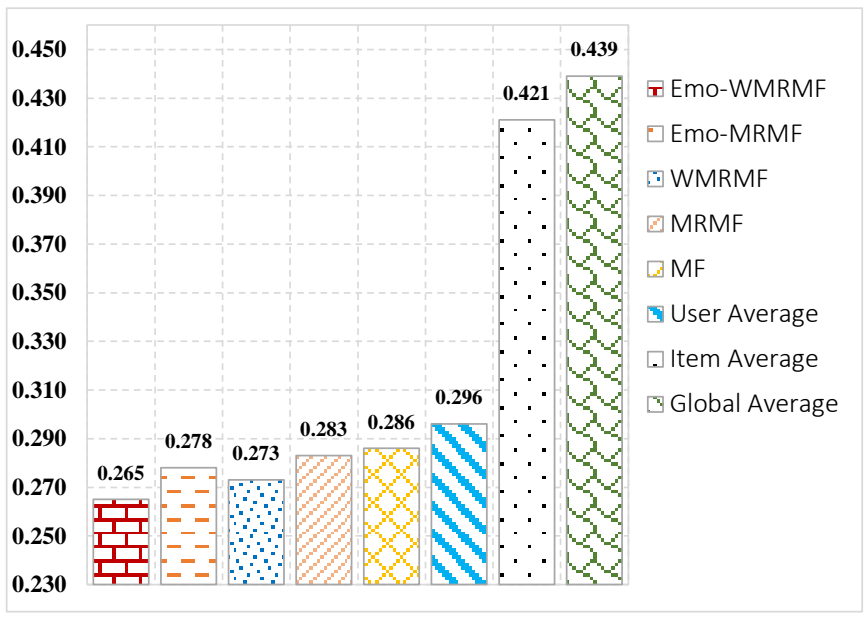

Fig. 4. RMSE Results on our Data Set.

Fig. 5 shows the RMSE results of Emotional Impact factor prediction for different values of parameter iteration. Indeed, the optimization of the model allows, of course, to predict performance, but also to predict the emotional impact of a student $s$ when carrying out a task $i$. When the number of 
iterations tends to around 320, the RMSE error in predicting emotional impact tends to around 0.232. This reveals the robustness of MF Models to predict all kinds of attributes of the application domain.

Fig. 5 also presents the RMSE evolution of the EmoWMRMF model. The emotional impact prediction curve decreases rapidly to 80 iteration steps and remains practically constant up to 320 . This shows that our model is robust already with an iteration number of 80 .

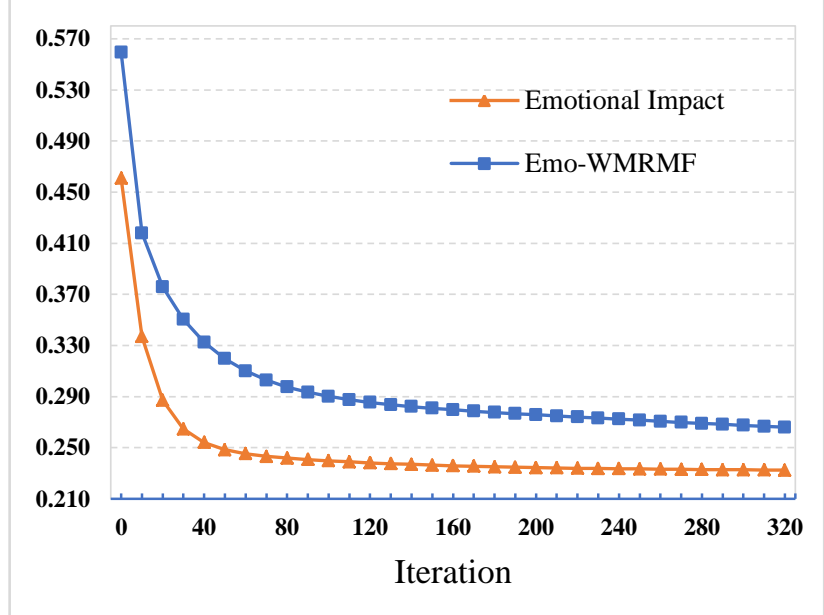

Fig. 5. RMSE with different Values of Iterations.

\section{CONCLUSION}

In this paper, we have proposed an approach which aims at providing a solution to student performance predicting problem in ITS by combining Multiple Linear Regression (modelling Emotional Impact) and a Weighted MultiRelational Matric Factorization model to take advantage of both student cognitive and emotional faculties. This approach takes into account not only the relationships that exist between students, tasks and skills, but also their emotions. Experimental results on a set of data collected in a general secondary school have shown that our approach makes it possible to more accurately predict student performance.

In future work, we plan to study the impact of the emotional state evolution during assessment sessions and analyze the effect of other emotional scales on student performance prediction results.

\section{REFERENCES}

[1] W. J. Hawkins, N. T. Heffernan et R. S. Baker, «Learning Bayesian Knowledge Tracing Parameters with a Knowledge Heuristic and Empirical Probabilities,» chez International Conference on Intelligent Tutoring Systems, 2014.

[2] J. Self, «The role of student models in learning environments,» chez Transactions of the Institute of Electronics, Information and Communication Engineers, 1994.

[3] A. Tack, T. François, A.-L. Ligozat et C. Fairon, «Modèles adaptatifs pour prédire automatiquement la compétence lexicale d'un apprenant de français langue étrangère,» chez JEP-TALN-RECITAL, Paris, France, 2016.

[4] M. H. Immordino-Yang, Emotions, Learning, and the Brain: Exploring the Educational Implications of Affective Neuroscience. The Norton Series on the Social Neuroscience of Education., New York: NY: W. W. Norton \& Company, 2016.
[5] C. Frasson et A. Trabelsi, «The Emotional Machine : A Machine Learning Approach to Online Prediction of User's Emotion and Intensity,» chez 10th IEEE International Conference on Advanced Learning Technologies, Sousse, Tunisia, 16 September 2010.

[6] R. W. Picard, «Affective Computing,» Cambridge : MIT Press, 1997.

[7] I. Magnisalis, S. Demetriadis et A. Karakostas, "Adaptive and Intelligent Systems for Collaborative Learning Support: A Review of the Field,» IEEE Transactions on Learning Technologies, vol. 4, pp. 5-20, 2011.

[8] R. Lukašenko, «Development of Student Model for Support of Intelligent Tutoring System Functions,» RTU Research Information System, 2012.

[9] H. Hamsa, S. Indiradevi et J. Kizhakkethottam, «Student Academic Performance Prediction Model Using Decision Tree and Fuzzy Genetic Algorithm,» chez Global Colloquium in Recent Advancement and Effectual Researches in Engineering, Science and Technology, 2016.

[10] R. Asif, A. Merceron, S. A. Ali et N. G. Haider, «Analyzing undergraduate students' performance using educational data mining,» Computers \& Education, vol. 113, pp. 177-194, October 2017.

[11] R. Hasan, S. Palaniappan, A. R. e. A. Raziff, S. Mahmood et K. U. Sarker, «Student Academic Performance Prediction by using Decision Tree Algorithm,» chez 4th International Conference on Computer and Information Sciences (ICCOINS), 2018.

[12] I. A. A. Amra et A. . Y. A. Maghari, «Students Performance Prediction Using KNN and Naïve Bayesian,» chez 8th International Conference on Information Technology (ICIT), 2017.

[13] F. Okubo, T. Yamashita, A. Shimada et H. Ogata, «A Neural Network Approach for Students' Performance Prediction,» chez the Seventh International Learning Analytics \& Knowledge Conference, 2017.

[14] J. Shana et T. Venkatachalam, «Identifying Key Performance Indicators and Predicting the Result from Student Data,» International Journal of Computer Applications, vol. 25, n \% 19, July 2011.

[15] H. Agrawal et H. Mavani, «Student Performance Prediction using Machine Learning,» International Journal of Engineering Research \& Technology (IJERT), vol. 4, n \% 103, March 2015.

[16] H. Lu et J. Yuan, «Student Performance Prediction Model Based on Discriminative Feature Selection,» 2018.

[17] H. Al-Shehri, A. Al-Qarni, L. Al-Saati, A. Batoaq, H. Badukhen, S. Alrashed, J. Alhiyafi et S. O. Olatunji, «Student Performance Prediction Using Support Vector Machine and K-Nearest Neighbor,» chez IEEE 30th Canadian Conference on Electrical and Computer Engineering (CCECE), 2017.

[18] S. Kadry, «Factors Affecting Students' Performance a Case of Private Colleges in Lebanon,» ScholArena, vol. 1, n \%11, October 2018.

[19] N. Thai-Nghe, L. Drumond, A. Krohn-Grimberghe et L. SchmidtThieme, «Recommender System for Predicting Student Performance,» chez 1st Workshop on Recommender Systems for Technology Enhanced Learning, 2010.

[20] E. Jembere, R. Rawatlal et A. W. Pillay, «Matrix Factorisation for Predicting Student Performance,» chez 7th World Engineering Education Forum (WEEF), 2017.

[21] N. Thai-Nghe et L. Schmidt-Thieme, «Multi-Relational Factorization Models for Student Modeling in Intelligent Tutoring Systems,» chez Seventh International Conference on Knowledge and Systems Engineering, 2015.

[22] K. A. Assielou, C. T. Haba, T. L. Kadjo, K. D. Yao et B. T. Gooré, «Multi-Relational and Social-Influence Model for Predicting Student Performance in Intelligent Tutoring Systems (ITS),» International Journal of Engineering and Advanced Technology, vol. 9, $\mathrm{n}^{\circ} \% 13$, pp. 2058-2066, 2020.

[23] L. Chen, G. Chen et F. Wang, «Recommender systems based on user reviews : the state of the art,» User Modeling and User-Adapted, vol. 25, $\mathrm{n}^{\circ} \% 12$, p. 99-154, 2015.

[24] P. Nedungadi et T. K. Smruthy, «Personalized Multi-relational Matrix Factorization Model for Predicting Student Performance,» 2016.

[25] G. Takacs, I. Pilaszy et B. Nemeth, «On the Gravity Recommendation System,» Proceedings of KDD Cup and Workshop, 2007. 
[26] C. Lippert, S. H. Weber, Y. Huang et V. Tresp, «Relation Prediction in Multi-Relational Domains using Matrix Factorization,» chez NIPS 2008 Workshop on Structured Input - Structured Output., 2008.

[27] «An Approach for Multi-Relational Data Context in Recommender Systems,» chez Asian Conference on Intelligent Information and Database Systems, 2017.

[28] H. Li, X. Diao, J. Cao et Q. Zheng, "Collaborative filtering recommendation based on all-weighted matrix factorization and fast optimization_2018,» Ieee Access, 2018.

[29] R. Pekrun, «Emotion and Achievement During Adolescence,» Child Development Perspectives, vol. 11, 2017.

[30] R. Pekrun et E. J. Stephens, «Academic emotions,» educational psychology handbook, p. 3-31, 2012.

[31] R. Pekrun, «The control-value theory of achievement emotions: Assumptions, corollaries, and implications for educational research and practice,» Educational Psychology Review, p. 315-341, 2006.

[32] R. Pekrun, T. Goetz, A. C. Frenzel et R. P. Perry, «Academic Emotions Questionnaire (AEQ). User's manual (2nd ed.).,» 2011.
[33] R. Pekrun, T. Goetz, A. C. Frenzel, P. Barchfeld et R. P. Perry, «Measuring emotions in students learning and performance: The Achievement Emotions Questionnaire (AEQ),» Contemporary Educational Psychology, vol. 36, p. 36-48, 2011.

[34] L. Myyry, T. Karaharju-Suvanto, M. Vesalainen, A.-M. Virtala, M. Raekallio, O. Salminen, K. Vuorensola et A. Nevgi, «Experienced academics' emotions related to assessment,» Assessment \& Evaluation in Higher Education, vol. 45, n \%11, pp. 1-13, 2019.

[35] R. Pekrun, Global and local perspectives on human affect: Implications of the control-value theory of achievement emotions, S. K. A. E. P. N. (. M Wosnitza, Éd., Cambridge, 2009, p. 97-115.

[36] M. Zeidner, Test anxiety in educational contexts: What I have learned so far, P. S. \&. R. P. (Eds.), Éd., San Diego: Academic Press, 2007.

[37] R. Pekrun, T. Goetz, A. Frenzel, P. Barchfeld et R. Perry, «Measuring emotions in students' learning and performance: The Achievement Emotions Questionnaire (AEQ).,» Contemporary Educational Psychology, vol. 36, p. 36-48.

[38] L. Isenman, «Emotion and Motivation,» chez Understanding Intuition, 2018, pp. 111-132. 